\title{
Impact of Hypoglycemia on Health-Related Quality of Life among Type 2 Diabetes: A Cross-Sectional Study in Thailand
}

\author{
Thongchai Pratipanawatr $\mathbb{D}^{1},{ }^{1}$ Bancha Satirapoj $\left(\mathbb{D},{ }^{2}\right.$ Boonsong Ongphiphadhanakul $\left(\mathbb{D},{ }^{3}\right.$ \\ Sompongse Suwanwalaikorn $\mathbb{D}^{\mathbb{D}},{ }^{4}$ and Wannee Nitiyanant ${ }^{5}$ \\ ${ }^{1}$ Department of Medicine, Faculty of Medicine, Srinagarind Hospital, Khon Kaen University, Khon Kaen 40002, Thailand \\ ${ }^{2}$ Department of Medicine, Phramongkutklao Hospital and College of Medicine, Bangkok 10400, Thailand \\ ${ }^{3}$ Department of Medicine, Faculty of Medicine, Ramathibodi Hospital, Mahidol University, Bangkok 10400, Thailand \\ ${ }^{4}$ Department of Medicine, Faculty of Medicine, King Chulalongkorn Memorial Hospital, Chulalongkorn University, \\ Bangkok 10330, Thailand \\ ${ }^{5}$ Department of Medicine, Siriraj Hospital, Bangkok, Thailand
}

Correspondence should be addressed to Thongchai Pratipanawatr; thongchaip@yahoo.com

Received 11 May 2019; Accepted 27 September 2019; Published 23 October 2019

Academic Editor: Virginia Boccardi

Copyright (C) 2019 Thongchai Pratipanawatr et al. This is an open access article distributed under the Creative Commons Attribution License, which permits unrestricted use, distribution, and reproduction in any medium, provided the original work is properly cited.

\begin{abstract}
Type 2 diabetes mellitus (T2DM) is one of the most common chronic diseases. Patients are generally advised lifestyle changes with antihyperglycemic agents prescribed. The major drawback of prescribing antihyperglycemic agents is the risk of hypoglycemia which subsequently impacts on health-related quality of life (HRQoL). This study is aimed at examining association between previous history of hypoglycemia and HRQoL. The study was a multicenter cross-sectional study, conducted from February 2013 to March 2015 at 5 tertiary care hospitals in Thailand (Srinagarind, Phramongkutklao, Ramathibodi, King Chulalongkorn Memorial, and Siriraj hospitals). The study population were males or females diagnosed with type 2 DM according to ADA criteria, 30 years of age or older, who had been treated with sulfonylurea (SU) monotherapy or SU and metformin combination for at least 6 months. Prespecified medical factors were extracted from medical records 12 months prior to patients' enrolment. The experience of hypoglycemia questionnaire was used to collect and measure severity of hypoglycemia experienced during the previous 6 months. HRQoL was assessed using the 3-level version of EuroQol-5-dimension (EQ-5D-3L) and visual analogue scale (EQ-VAS) questionnaires. Of 659 eligible patients surveyed, 202 patients (30.65\%) had experienced symptoms of hypoglycemia. HRQoL was significantly lower among patients reporting at least one of hypoglycemic symptoms, measured by EQ-VAS scores (mean \pm SD; $73.66 \pm 13.18,73.56 \pm 15.10$, or $68.93 \pm 14.76$ vs. $77.01 \pm 13.02$, one-way ANOVA; $p=0.006$ ) and EQ-5D-3L index scores $(0.62 \pm 0.47,0.68 \pm 0.38$, or $0.58 \pm 0.51$ vs. $0.79 \pm 0.31$, one-way ANOVA; $p<0.001)$ for mild, moderate, or severe/very severe hypoglycemic patients compared with patients without hypoglycemic symptoms. After adjusting for confounding factors in a multiple linear regression model, patients with hypoglycemic symptoms either mild, moderate, or severe/very severe demonstrated significantly higher impairment for EQ-VAS and EQ-5D indexes than those who did not experience hypoglycemic symptoms. In conclusion, our study showed decreased HRQoL determined by EQ-5D and EQ-VAS in patients reporting symptoms of hypoglycemia compared with patients not reporting hypoglycemic symptoms, relative to severity of hypoglycemia.
\end{abstract}

\section{Introduction}

Type 2 diabetes mellitus (T2DM) is a chronic and progressive disease with serious long-term micro- and macrovascular complications [1] resulting in an increase in morbidity, mor- tality, healthcare costs, and a decrease in HRQoL. There are several treatments for diabetes, with the most common treatment regimen being the prescription of oral antihyperglycemic agents and insulin therapy which consequently induce hypoglycemia. The most prominent hypoglycemia inducers 
are sulfonylureas and insulin therapy. Hypoglycemia can be a major barrier to achieving treatment goals and has been shown to increase risks of cardiovascular events [2], dementia [3], fall-related fracture [4], and "defensive eating" which contributes to weight gain. In addition, patients' apprehension about hypoglycemia can also be a major implication in disease management [5]. The particular impact of hypoglycemia on health-related quality of life (HRQoL) has often been demonstrated in observational studies $[6,7]$, suggesting the indirect correlation between hypoglycemia induced from diabetes treatments on HRQoL. Nonetheless, the hypoglycemic symptoms seen in diabetic patients may also be influenced by other factors including old age, weight gain, comorbidity and cardiovascular disease, gender differences, types of antihyperglycemic agents taken such as metformin, concerns about hypoglycemia, and overall patients' satisfaction, which in-turn may also affect patients' quality of life. Therefore, the association of hypoglycemia with HRQoL is inconclusive as there are other confounding factors involved. This study is aimed at examining the association between previous history of hypoglycemia and HRQoL in Thai T2DM patients.

\section{Materials and Methods}

2.1. Study Design and Setting. This study was a multicenter cross-sectional study conducted in patients who have been treated with sulfonylurea (SU) monotherapy or SU and metformin combination therapy (SU+MET) for at least 6 months prior to the study enrolment in 5 tertiary care hospitals in Thailand (i.e., Srinagarind, Phramongkutklao, Ramathibodi, King Chulalongkorn Memorial, and Siriraj hospitals). The study was conducted from February 2013 to March 2015. This study was approved by the Ethics Committee of each hospital. The potential patient medical charts were retrospectively reviewed to check if they meet inclusion and exclusion criteria. The patients satisfying the selection criteria were enrolled in the study after providing written informed consent to participate in the study.

2.2. Participants. The patients who were diagnosed with T2DM according to American Diabetes Association (ADA) criteria [8], 30 years of age or older, and treated with SU monotherapy or SU+MET for at least 6 months before enrolment by an endocrinologist, cardiologist, nephrologist, or family practitioner were included, whereas the patients who were pregnant; have Type $1 \mathrm{DM}$; required daily concomitant insulin; received other oral hypoglycemic agents, except for SU or SU combined with metformin; were participating in other clinical trial studies; and were not able to complete the study questionnaires were excluded from this study.

2.3. Study Measurements and Study Data Collection. A standardized data collection form was developed to record data of clinical and laboratory assessment of patients from medical records that were completed by physicians or personnel who had been trained. Prespecified medical data from charts were extracted for the 12-month period prior to the patient enrolment date. On the day of enrolment, participating patients were subjected to a standard blood draw after overnight fasting for measurements of $\mathrm{HbA}_{1 \mathrm{c}}$, fasting plasma glucose, serum creatinine, total cholesterol, triglycerides, HDLcholesterol, and LDL-cholesterol. In addition, each patient's body weight, blood pressure, and waist circumference were measured and recorded. Alcohol consumption and regular physical activity were also assessed.

Experience of hypoglycemia during the previous 6 months was assessed with the questionnaire that was developed by Merck Sharp \& Dohme (MSD) (see the Experience of Low Blood Sugar (Hypoglycemia) questionnaire in Supplementary Material 1). The hypoglycemia symptoms were stratified by severity (from none, mild, moderate, and severe to very severe). The patients were subsequently classified according to having experienced hypoglycemia or not (yes/no) and according to the maximum severity of hypoglycemic episodes experienced.

HRQoL was assessed by using the Thai language 3-level version of EuroQol 5-dimension (EQ-5D-3L) questionnaire which is a standardized instrument for measuring the general health outcome and is a recommended utility method because it has acceptable feasibility and validity in Thailand [9]. The EQ-5D contains 5 items to be answered using a 3-point rating scale plus a visual analogue scale (VAS) from 0 (worst imaginable health state) to 100 (best imaginable health state) [10]. The EQ-5D measures 5 dimensions of health: mobility, self-care, usual activities, pain/discomfort, and anxiety/depression. The EQ-5D index score was calculated using the coefficient from the "Social tariff for EuroQol" [11].

The treatment satisfaction was evaluated by using the 14-item treatment satisfaction questionnaire for medication (TSQM, version 1.4) [12]. The satisfaction score per dimension was calculated ranging from 0 to 100 , with a lower score expressing a better treatment satisfaction. Worry about hypoglycemia and self-reported adherence were assessed by the Worry Scale of HFS II $[13,14]$ and the Self-Report Adherence and Barriers Questionnaire [15], respectively. The questionnaires were previously translated from English to Thai and used in a diabetes study (the Asia-Pacific RealLife Effectiveness and Care Patterns of Diabetes Management (AP RECAP-DM) study) [16]. The authors also reviewed and verified the accuracy of the translations prior to use in this study.

2.4. Sample Size. We estimated the sample size in order to conduct our survey of HRQoL in hypoglycemia patients using the following formula [17]: $n=\left(Z^{2} \times P(1-P)\right) / d^{2}$. The expected prevalence of hypoglycemia was $36 \%$ which was reported in the AP RECAP-DM study (Poster No. P44, $7^{\text {th }}$ IDF Western Pacific Region Congress, Wellington, New Zealand, March 30-April 3, 2008). The sample size was calculated assuming the statistic corresponding level of confidence of $95 \%(Z, 1.96)$, hypoglycemia proportion of 0.36 , and a desired margin of error $(d)$ of $\pm 3.5 \%$ to $4 \%$. Therefore, we planned to study HRQoL in approximately 600 to 723 eligible patients.

2.5. Statistical Analysis. Descriptive statistics were used to report demographic characteristics of patients and clinical 


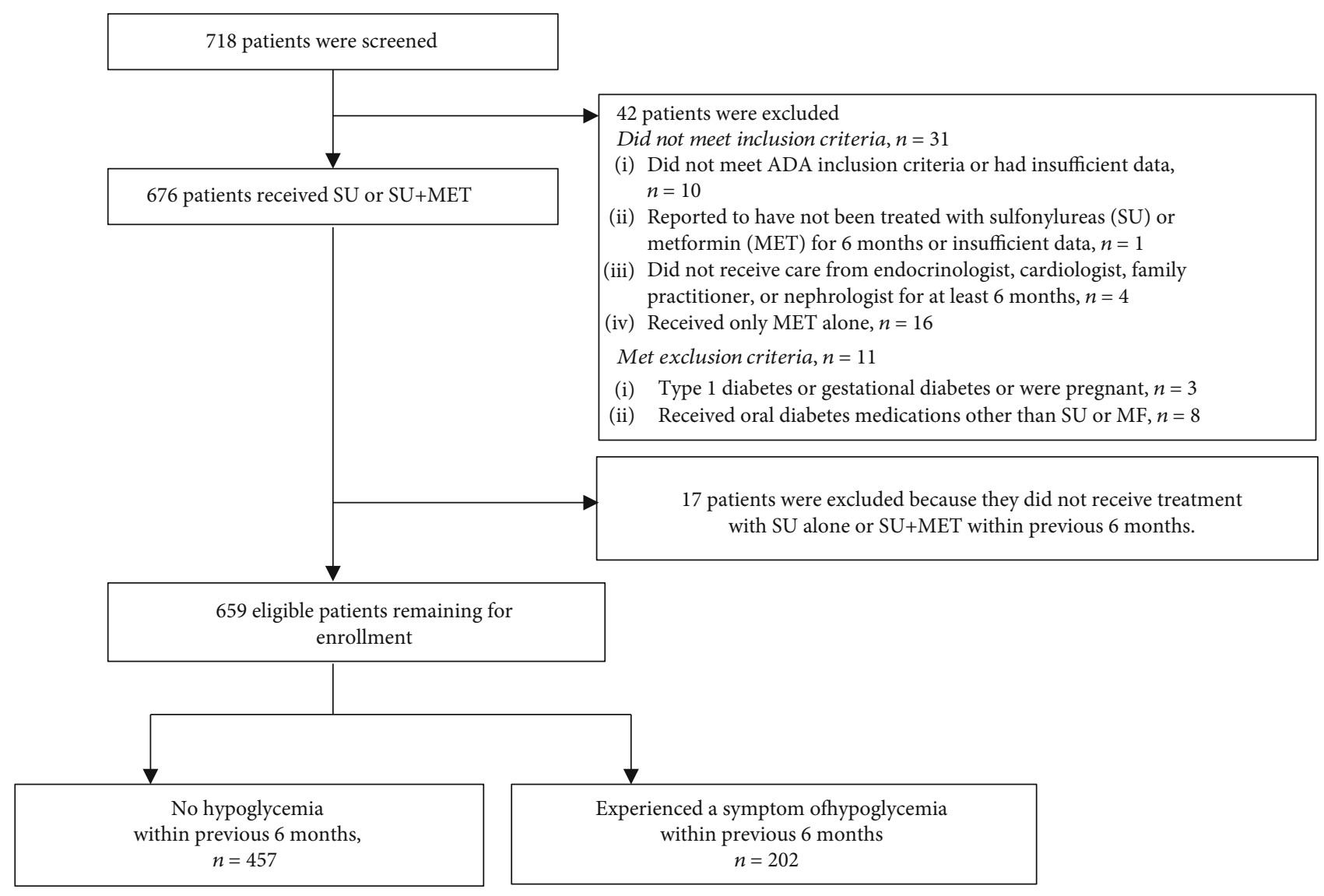

Figure 1: Participant flow.

characteristics, including patient sociodemographic, clinical, and laboratory test results. The Shapiro-Wilk test was used for checking normality of the continuous data. If the data distribution is normal, the data will be presented by the mean $( \pm S D)$. If the data significantly deviate from a normal distribution ( $p$ value $<0.05$ ), the data will be presented by the median (IQR). The demographic characteristics, laboratory results, and HRQoL of patients with and without reported hypoglycemic symptoms were compared using the Chisquared test or Fisher's exact test for categorical variables and independent sample $t$-test or Mann-Whitney $U$ test for continuous variables.

One-way analysis of variance (one-way ANOVA) was conducted to evaluate differences in EQ-VAS scores by severity of hypoglycemia episodes and evaluated differences in EQ-5D-3L index scores by episode severity of hypoglycemia. Multivariate linear regression, adjusting for potential confounders, was used to estimate the effect of hypoglycemia on HRQoL (EQ-VAS and EQ-5D-index scores). The technique for selecting the covariates in the multivariate regression analyses was that based on the directed acyclic graph (DAG). Multivariate relationships were conceptualized using directed acyclic graphs and minimum sets of adjustment variables to obtain unbiased estimates of total and direct effects of various exposure variables on occurrence of hypoglycemia, treatment compliance, treatment satisfaction, quality of life, worry about hypoglycemia, and fear of weight gain compatible with the conceptual graph identified.

Directed acyclic graphs were constructed using DAGitty software (version 2.3) [18]. All test statistics applied a $p$ value less than 0.05 as statistically significant. The study data analyses were performed using STATA release 14.1 (StataCorp, College Station, TX).

\section{Results}

3.1. Participants. Participant flow is shown in Figure 1 . Approximately 30\% (202 of 659) of type 2 diabetes patients experienced symptoms of hypoglycemia in 6 months prior to the survey.

Compared to those who did not experience symptoms of hypoglycemia, patients who did experience symptoms of hypoglycemia were significantly younger (mean $\pm \mathrm{SD}$, $63.94 \pm 10.65$ years vs. $66.20 \pm 9.59$ years, $t$-test; $p=0.008)$ and less commonly took regular physical activities (no regular activity rate; $34.3 \%$ vs. $42.8 \%$, Chi-squared test; $p=0.038$ ). In addition, the patients experiencing hypoglycemia were more commonly on a low-sugar diet higher than those who did not experience symptoms of hypoglycemia ( $57.7 \%$ vs. $47.6 \%$, Chi-squared test; $p=0.018)$ and were more commonly nondrinkers $(74.5 \%$ vs. $69.6 \%$, Chi-squared test; $p=0.030)$. 
TABle 1: Patient demographics by occurrence of hypoglycemia in previous 6 months.

\begin{tabular}{|c|c|c|c|}
\hline Variables & No hypoglycemia $(n=457)$ & Hypoglycemia $(n=202)$ & $p$ value \\
\hline Male, $n(\%)$ & $228(50.8)$ & $93(46.0)$ & $0.272^{\mathrm{a}}$ \\
\hline Age (years) & $66.20(9.59)$ & $63.94(10.65)$ & $0.008^{\mathrm{b} \dagger}$ \\
\hline Duration of DM (years), median (IQR) & $10(5,15)$ & $10(6,15)$ & $0.531^{\mathrm{c}}$ \\
\hline Height (cms) & $160.5(8.4)$ & $160.3(9.3)$ & $0.746^{\mathrm{b}}$ \\
\hline Body weight (kgs) & $66.5(12.9)$ & $65.2(14.5)$ & $0.134^{\mathrm{b}}$ \\
\hline Did not take physical regular activity, $n(\%)$ & $156(34.3)$ & $86(42.8)$ & $0.038^{\mathrm{a} \dagger}$ \\
\hline \multicolumn{4}{|l|}{ Alcohol consumption, $n(\%)$} \\
\hline Never & $318(69.6)$ & $149(74.5)$ & \multirow[t]{4}{*}{$0.030^{\mathrm{a} \dagger}$} \\
\hline Occasionally & $116(25.4)$ & $45(22.5)$ & \\
\hline Daily & $1(0.2)$ & $3(1.5)$ & \\
\hline Unknown & $22(4.8)$ & $3(1.5)$ & \\
\hline Low-sugar diet, $n(\%)$ & $214(47.6)$ & $116(57.7)$ & $0.018^{\mathrm{a} \dagger}$ \\
\hline \multicolumn{4}{|l|}{ Weight change in previous 12 months } \\
\hline No change & $87(19.3)$ & $42(21.1)$ & \multirow[t]{4}{*}{$0.798^{\mathrm{a}, \beta}$} \\
\hline Gained & $158(35.0)$ & $65(32.7)$ & \\
\hline Lost & $192(42.5)$ & $85(42.7)$ & \\
\hline Unknown & $15(3.3)$ & $7(3.5)$ & \\
\hline Waist circumference $(\mathrm{cm})$ & $92.4(10.1)$ & $91.0(11.7)$ & $0.119^{\mathrm{b}}$ \\
\hline SBP (mmHg) & $135.7(17.1)$ & $133.5(17.6)$ & $0.128^{\mathrm{b}}$ \\
\hline DBP (mmHg) & $74.5(10.2)$ & $73.4(9.8)$ & $0.186^{\mathrm{b}}$ \\
\hline \multicolumn{4}{|l|}{ Hypoglycemic agents, $n(\%)$} \\
\hline Sulfonylurea (SU) & $93(20.3)$ & $45(22.3)$ & \multirow[t]{2}{*}{$0.604^{\mathrm{a}}$} \\
\hline Combination of SU and metformin & $364(79.7)$ & $157(77.7)$ & \\
\hline Macro- and/or microvascular complications, $n(\%)$ & $99(21.7)$ & $38(18.8)$ & $0.407^{\mathrm{a}}$ \\
\hline
\end{tabular}

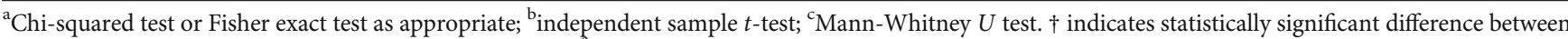
the occurrence of hypoglycemia groups $(p$ value $<0.05) .{ }^{\beta}$ Only known data used for analysis. Data are the mean \pm standard deviation $(\mathrm{SD})$ unless otherwise specified. Abbreviation: SD: standard deviation; IQR: interquartile range.

There were no significant differences in waist circumference, systolic and diastolic blood pressure, micro-/macrovascular complications, and hypoglycemic agents used between the patients with hypoglycemia and without hypoglycemia. In addition, there were no significant differences in marital status, education, or having parents with diabetes mellitus (data not shown). There were no significant differences between patients taking SU versus patients taking SU+MET in terms of hypoglycemia symptoms experienced. The patient demographics by occurrence of hypoglycemia in the previous 6 months are shown in Table 1. Furthermore, there were also no significant differences in respect to the laboratory test results between patients who did and did not experience hypoglycemic symptoms (Table 2 ).

3.2. Hypoglycemia and EQ-VAS and EQ-5D Index Scores. HRQoL was significantly lower among patients reporting at least one of hypoglycemic symptoms either mild, moderate, or severe/very severe symptoms, as measured by EQ-VAS scores (mean $\pm \mathrm{SD} ; 73.66 \pm 13.18,73.56 \pm 15.10$, or $68.93 \pm$ 14.76 vs. $77.01 \pm 13.02$, one-way ANOVA; $p=0.006)$ and EQ-5D index scores $(0.62 \pm 0.47,0.68 \pm 0.38$, or $0.58 \pm 0.51$ vs. $0.79 \pm 0.31$, one-way ANOVA; $p<0.001)$ compared to patients reporting no symptoms as shown in Table 3.
3.3. Hypoglycemia and EQ-5D Subscores. There was significant association between quality of life in each domain and severity of hypoglycemic episodes experienced as shown in Table 4.

3.4. Association between Severity of Hypoglycemic Episodes and HRQoL. The results show that higher severity of hypoglycemic episodes experienced, use of SU+MET, higher worry about hypoglycemia scores, weight changes (either lost or gained), and higher fear of weight gain scores were significant factors lowering EQ-5D-3L index score while being male significantly increased the score. The patients with higher severity of hypoglycemic symptoms were negatively associated with HRQoL as measured by EQ-5D-3L index score than those who did not have hypoglycemia (adjusted mean difference; for mild: -0.156 (95\% CI: $-0.225,-0.087$ ), moderate: -0.096 (CI: $-0.183,-0.008)$, and severe/very severe: -0.198 (CI: $-0.373,-0.023)$ when compared to those who have not experienced hypoglycemia).

For HRQoL, as measured by EQ-VAS scores, higher severity of hypoglycemic episodes experienced and higher worry about hypoglycemia were significant factors lowering EQ-VAS score while higher overall satisfaction was related with a higher EQ-VAS score. The patients with higher 
TABLE 2: Laboratory test results at enrolment by occurrence of hypoglycemia in the previous 6 months.

\begin{tabular}{|c|c|c|c|}
\hline Variables & No hypoglycemia $(n=457)$ & Hypoglycemia $(n=202)$ & $p$ value \\
\hline $\mathrm{HbA}_{1 \mathrm{c}}(\%)$ & $7.29(1.28)$ & $7.17(1.31)$ & $0.247^{\mathrm{a}}$ \\
\hline FPG (mg/dL) & $145.6(44.6)$ & $139.4(39.7)$ & $0.086^{\mathrm{a}}$ \\
\hline Serum creatinine $(\mathrm{mg} / \mathrm{dL})$ & $1.26(1.08)$ & $1.23(0.89)$ & $0.767^{\mathrm{a}}$ \\
\hline Total cholesterol (mg/dL) & $167.8(38.5)$ & $170.3(36.1)$ & $0.581^{\mathrm{a}}$ \\
\hline HDL-cholesterol (mg/dL) & $50.4(20.4)$ & $51.1(15.0)$ & $0.784^{\mathrm{a}}$ \\
\hline LDL-cholesterol (mg/dL) & $97.7(33.9)$ & $98.1(32.4)$ & $0.912^{\mathrm{a}}$ \\
\hline Triglycerides (mg/dL), median (IQR) & $133(92,179)$ & $132.5(91.5,180)$ & $0.632^{\mathrm{b}}$ \\
\hline Urinary albumen (mg/g), median (IQR) & $10.8(4.7,30.3)$ & $7.3(2.3,46.4)$ & $0.573^{\mathrm{b}}$ \\
\hline
\end{tabular}

${ }^{a}$ Independent sample $t$-test. ${ }^{\mathrm{b}}$ Mann-Whitney $U$ test. Data are the mean \pm standard deviation $(\mathrm{SD})$ unless otherwise specified. Abbreviation: $\mathrm{HbA}_{1 \mathrm{c}}=$ hemoglobin Alc; FPG = fasting plasma glucose .

TABLe 3: Patient EQ-5D-VAS and EQ-5D-index scores according to severity of hypoglycemia.

\begin{tabular}{|c|c|c|c|c|}
\hline HRQoL & Maximum severity of hypoglycemic episodes experienced & Mean (SD) & $95 \% \mathrm{CI}$ & $p$ value* \\
\hline \multirow{4}{*}{ EQ-5D-VAS score } & None & $77.01(13.02)$ & $75.77,78.22$ & \multirow{4}{*}{0.006} \\
\hline & Mild & $73.66(13.18)$ & $71.27,76.06$ & \\
\hline & Moderate & $73.56(15.10)$ & $70.37,76.45$ & \\
\hline & Severe/very severe & $68.93(14.76)$ & $62.18,75.68$ & \\
\hline \multirow{4}{*}{ EQ-5D index score } & None & $0.79(0.31)$ & $0.76,0.82$ & \multirow{4}{*}{$<0.001$} \\
\hline & Mild & $0.62(0.47)$ & $0.56,0.69$ & \\
\hline & Moderate & $0.68(0.38)$ & $0.60,0.76$ & \\
\hline & Severe/very severe & $0.58(0.51)$ & $0.40,0.75$ & \\
\hline
\end{tabular}

*One-way ANOVA. EQ-VAS score ranges from 0 to 100, with a higher score indicating better quality of life. EQ-5D index score was calculated using the coefficient from "Social tariff for EuroQol" [11].

TABle 4: Patient EQ-5D-3L levels by maximum severity of hypoglycemic episodes.

\begin{tabular}{|c|c|c|c|c|c|c|}
\hline \multirow{2}{*}{ Domain } & \multirow{2}{*}{ Maximum severity of hypoglycemic episodes } & \multirow{2}{*}{$n$} & \multicolumn{3}{|c|}{ Level of perceived problem, $n(\%)$} & \multirow{2}{*}{$p$ value* } \\
\hline & & & No problem & Some problem & Extreme problems & \\
\hline \multirow{4}{*}{ Mobility } & None & 457 & $297(65.0)$ & $140(30.6)$ & $20(4.4)$ & \multirow{4}{*}{$0.002^{\dagger}$} \\
\hline & Mild & 119 & $52(43.7)$ & $55(46.2)$ & $12(10.1)$ & \\
\hline & Moderate & 67 & $35(52.2)$ & $28(41.8)$ & $4(6.0)$ & \\
\hline & Severe/very severe & 15 & $9(60.0)$ & $5(33.3)$ & $1(6.7)$ & \\
\hline \multirow{4}{*}{ Self-care } & None & 457 & $383(83.8)$ & $71(15.5)$ & $3(0.7)$ & \multirow{4}{*}{$0.001^{\dagger}$} \\
\hline & Mild & 119 & $80(67.2)$ & $35(29.4)$ & $4(3.4)$ & \\
\hline & Moderate & 67 & $54(80.6)$ & $12(17.9)$ & $1(1.5)$ & \\
\hline & Severe/very severe & 15 & $10(66.7)$ & $4(26.7)$ & $1(6.7)$ & \\
\hline \multirow{4}{*}{ Usual activities } & None & 457 & $368(80.5)$ & $80(17.5)$ & $9(2.0)$ & \multirow{4}{*}{$0.001^{\dagger}$} \\
\hline & Mild & 119 & $76(63.9)$ & $39(32.8)$ & $4(3.4)$ & \\
\hline & Moderate & 67 & $48(71.6)$ & $16(23.9)$ & $3(4.5)$ & \\
\hline & Severe/very severe & 15 & $9(60.0)$ & $4(26.7)$ & $2(13.3)$ & \\
\hline \multirow{4}{*}{ Pain/discomfort } & None & 457 & $265(58.1)$ & $166(36.4)$ & $25(5.5)$ & \multirow{4}{*}{$0.008^{\dagger}$} \\
\hline & Mild & 119 & $50(42.4)$ & $54(45.8)$ & $14(11.9)$ & \\
\hline & Moderate & 67 & $28(41.8)$ & $32(47.8)$ & $7(10.4)$ & \\
\hline & Severe/very severe & 15 & $6(40.0)$ & $7(46.7)$ & $2(13.3)$ & \\
\hline \multirow{4}{*}{ Anxiety/depression } & None & 457 & $341(75.1)$ & $102(22.5)$ & $11(2.4)$ & \multirow{4}{*}{$<0.001^{\dagger}$} \\
\hline & Mild & 119 & $64(54.2)$ & $42(35.6)$ & $12(10.2)$ & \\
\hline & Moderate & 67 & $35(52.2)$ & $26(38.8)$ & $6(9.0)$ & \\
\hline & Severe/very severe & 15 & $7(46.7)$ & $4(26.7)$ & $4(26.7)$ & \\
\hline
\end{tabular}

${ }^{*}$ Chi-squared test. Numbers may not add up to the total because of missing data. $\dagger$ indicates statistically significant difference $(p<0.05)$. 
TABLE 5: Factors associated with health-related quality of life measured by EQ-5D-3L index and EQ-VAS scores from multivariate regression analyses.

\begin{tabular}{|c|c|c|c|c|}
\hline Variables* & $\begin{array}{c}\text { Adjusted mean difference (95\% CI) } \\
\text { of EQ-5D-3L index score }\end{array}$ & $p$ value & $\begin{array}{c}\text { Adjusted mean difference }(95 \% \mathrm{CI}) \\
\text { of EQ-5D-3L VAS score }\end{array}$ & $p$ value \\
\hline \multicolumn{5}{|l|}{ Hypoglycemia } \\
\hline None & Reference & \multirow[t]{4}{*}{$<0.001^{\dagger}$} & Reference & \multirow[t]{4}{*}{$0.013^{\dagger}$} \\
\hline Mild & $-0.156(-0.225,-0.087)$ & & $-2.92(-5.61,-0.23)$ & \\
\hline Moderate & $-0.096(-0.183,-0.008)$ & & $-3.17(-6.59,-0.25)$ & \\
\hline Severe/very severe & $-0.198(-0.373,-0.023)$ & & $-7.89(-14.71,-1.07)$ & \\
\hline Age $\geq 60$ years & $-0.057(-0.118,0.003)$ & 0.064 & $-0.32(-2.63,1.99)$ & 0.784 \\
\hline Male & $0.108(0.054,0.161)$ & $<0.001^{\dagger}$ & $1.48(-0.57,3.53)$ & 0.156 \\
\hline Vascular complication & $-0.020(-0.046,0.087)$ & 0.547 & $-2.24(-4.77,0.29)$ & 0.082 \\
\hline \multicolumn{5}{|l|}{ Treatment } \\
\hline SU alone & Reference & \multirow[t]{2}{*}{$0.049^{\dagger}$} & Reference & \multirow[t]{2}{*}{0.127} \\
\hline SU+MET & $-0.067(-0.133,0.000)$ & & $1.97(-0.56,4.51)$ & \\
\hline \multicolumn{5}{|l|}{ Weight change } \\
\hline None & Reference & \multirow[t]{3}{*}{$0.003^{\dagger}$} & Reference & \multirow[t]{3}{*}{0.809} \\
\hline Gain & $-0.116(-0.189,-0.042)$ & & $0.83(-2.05,3.71)$ & \\
\hline Loss & $-0.119(-0.190,-0.048)$ & & $0.88(-1.92,3.67)$ & \\
\hline Adherence to medication & $-0.018(-0.071,0.034)$ & 0.490 & $-0.45(-2.49,1.60)$ & 0.668 \\
\hline Worry about hypoglycemia/unit score (0 to 4$)$ & $-0.059(-0.103,-0.015)$ & $0.009^{\dagger}$ & $-3.14(-4.83,-1.46)$ & $<0.001^{\dagger}$ \\
\hline Fear of weight gain/unit score (0 to 4$)$ & $-0.070(-0.099,-0.041)$ & $<0.001^{\dagger}$ & $-0.73(-1.85,0.39)$ & 0.203 \\
\hline Overall satisfaction/unit score (0 to 100$)$ & $0.0016(-0.0008,0.0039)$ & 0.184 & $0.103(0.014,0.193)$ & $0.024^{\dagger}$ \\
\hline
\end{tabular}

${ }^{*}$ Variables included in multivariate regression analyses. Wald test, $p=p$ value. $\dagger$ indicates statistical significance at $p<0.05$.

severity of hypoglycemic episodes experienced symptoms also demonstrated significantly higher impairment for EQVAS score with adjusted mean difference of -2.92 (95\% CI: -5.61, -0.23), -3.17 (95\% CI: -6.59, 0.25), and -7.89 (95\% CI: $-14.71,-1.07)$ for mild, moderate, and severe/very severe hypoglycemia symptoms when compared to those who have not experienced hypoglycemia. Table 5 presents the adjusted mean differences of EQ-5D-3L index and EQ-VAS scores of each factor.

\section{Discussion}

In this study of Thai patients with T2DM treated with SU alone or SU+MET, symptoms of hypoglycemia were reported by approximately $30.65 \%$ of patients and only $6.59 \%$ reported severe or very severe events. The proportion of patients reporting hypoglycemic symptoms reported by United State (US) patients (63\% during 6 months [19]) and European patients (38\% during the past year [20] and $73 \%$ during 3 months [21]) treated with oral antihyperglycemic agents were higher than our study. However, a study in which we found a high prevalence of any form of reported hypoglycemia was in an urban African-American population with type 2 diabetes which reported lower proportion (16\%) for those using oral agents alone [22].

Our results demonstrated that patients with hypoglycemic symptoms reported a decrease in EQ-5D index and EQ-5 VAS scores relative to patients who did not, even after adjusting for a number of confounders. This is similar to the results published in Marrett et al. [23], Williams et al. [6],
Gilet et al. [24], and Shi et al. [25] which also used the EQ-5D and observed that self-reported hypoglycemia was associated with decrease in adjusted EQ-5D scores [23]. Our results also showed a relationship between the severity of hypoglycemia and decrease in EQ-5D index and EQVAS, emphasizing the effect of hypoglycemia on quality of life. Patients who experienced hypoglycemic symptoms reported decreases in all $5 \mathrm{EQ}-5 \mathrm{D}$ domains. There are more limitation on self-care domain in our study, which were different from the study by Williams et al., in which self-care activities were not significantly different between the hypoglycemia severity groups [6]. These study results are similar to the results of Marrett et al. [23], which also used EQ-5D and found that the adjusted mean EQ-5D index score (reference $=$ no hypoglycemia) was significantly 0.045 lower for those reporting any hypoglycemic symptoms and by symptom severity: mild 0.009 , moderate 0.055 , severe 0.131 , and very severe 0.208 [23]. Our results also show that the worry about hypoglycemia decreases both EQ-5D index and EQ-VAS scores. Therefore, therapies, patient monitoring rationales, or patient education programs that minimize the frequency and severity of hypoglycemia and worry about hypoglycemia would likely increase the diabetic patients' quality of life.

The current results should be considered within the context of several limitations. Hypoglycemia was determined by a patient-reported low plasma glucose questionnaire, crosssectional survey. The hypoglycemia data were not verified against clinician diagnoses or chart reviews nor were reports of low blood sugar confirmed by blood glucose monitoring. 
The future studies of quality of life at the time or immediately after the hypoglycemia symptom confirmation or when the hypoglycemia is confirmed by blood sugar monitoring are needed. In addition, this study is limited by its crosssectional design. Future studies with more rigorous designs, such as prospective cohort studies, are also needed to confirm the impact of hypoglycemia and to compare within the individual patients.

Additional consideration is that EQ-5D index scores were calculated using the coefficient from the "Social tariff for EuroQol" [11], which is based on the perceptions of a sample drawn from the UK population. Quality-of-life perceptions may be different in the Thai population from those in a UK population. However, EQ-VAS score which can directly quantify overall quality of life showed similar results to EQ-5D index score. Although a study that compared between EQ-5D-5L and EQ-5D-3L supported the convergent validity and test-retest reliability of both the $3 \mathrm{~L}$ and $5 \mathrm{~L}$ in diabetes patients, the authors recommend to use the $5 \mathrm{~L}$ due to being more promising in terms of a lower ceiling, more discriminatory power, and higher preference by the respondents compared to the $3 \mathrm{~L}$ [26]. Therefore, the future study in Thai patients with EQ-5D-5L is recommended.

\section{Conclusions}

In this survey of Thai patients with T2DM who were treated with SU alone or SU+MET, approximately one-thirds reported experiencing hypoglycemic symptoms. The results from the study showed significant decrement of HRQoL as determined by the EQ-5D index scores and the EQ-VAS in the patient-reported symptoms of hypoglycemia compared with patients who did not report those symptoms, relative to severity of hypoglycemia. Even after adjusting for a number of variables, patients who experienced hypoglycemic symptoms still continued to report a decrease in the HRQoL.

\section{Data Availability}

The patient-level data used to support the findings of this study have not been made publicly or shared with the third parties due to the institutes/hospitals' Human Research Ethics Committee regulations.

\section{Conflicts of Interest}

The authors declare that there is no conflict of interest regarding the publication of this paper. Although MSD (Thailand) Ltd. supported the study funding, the study was conducted and the study results were interpreted without the influence of the pharmaceutical company.

\section{Authors' Contributions}

All authors contributed equally to the study conduct. TP mainly drafted the article. All other authors reviewed the literatures and revised it critically. All authors read and approved the manuscript and met the criteria for authorship.

\section{Acknowledgments}

The authors gratefully acknowledge the contributions of the participating patients and general practitioners. They would like to thank Alan Geater and Walailuk Jitpiboon, Epidemiology Unit, Faculty of Medicine, Prince of Songkhla University, for statistical analysis. Merck Sharp and Dohme (Thailand) Ltd. supported for the study funding (e.g., cost of materials, data management, data analysis, and cost of publication).

\section{Supplementary Materials}

The study questionnaires (i.e., Experience of Low Blood Sugar, Worry Scale of HFS II, TSQM, version 1.4) were translated from English to Thai by the Psychology Department, Faculty of Liberal Arts, Thammasat University. The translations were done by forth and back translation. Content validity was checked using double-blind fashion. The questionnaires were tested by 15 diabetic patients, and the average duration to complete the questionnaire was 41.25 minutes. (Supplementary Materials)

\section{References}

[1] M. L. Pedersen, J. L. Jacobsen, and A. R. Lynge, "Micro-and macrovascular complications among Greenlanders and Danes with type 2 diabetes mellitus in Nuuk, Greenland," International Journal of Circumpolar Health, vol. 69, no. 2, pp. 195207, 2010.

[2] S. S. Johnston, C. Conner, M. Aagren, D. M. Smith, J. Bouchard, and J. Brett, "Evidence linking hypoglycemic events to an increased risk of acute cardiovascular events in patients with type 2 diabetes," Diabetes Care, vol. 34, no. 5, pp. 1164-1170, 2011.

[3] R. A. Whitmer, A. J. Karter, K. Yaffe, C. P. Quesenberry Jr., and J. V. Selby, "Hypoglycemic episodes and risk of dementia in older patients with type 2 diabetes mellitus," JAMA, vol. 301, no. 15, pp. 1565-1572, 2009.

[4] S. S. Johnston, C. Conner, M. Aagren, K. Ruiz, and J. Bouchard, "Association between hypoglycaemic events and fall-related fractures in Medicare-covered patients with type 2 diabetes," Diabetes, Obesity \& Metabolism, vol. 14, no. 7, pp. 634-643, 2012.

[5] D. Wild, R. von Maltzahn, E. Brohan, T. Christensen, P. Clauson, and L. Gonder-Frederick, "A critical review of the literature on fear of hypoglycemia in diabetes: implications for diabetes management and patient education," Patient Education and Counseling, vol. 68, no. 1, pp. 10-15, 2007.

[6] S. A. Williams, M. F. Pollack, and M. DiBonaventura, "Effects of hypoglycemia on health-related quality of life, treatment satisfaction and healthcare resource utilization in patients with type 2 diabetes mellitus," Diabetes Research and Clinical Practice, vol. 91, no. 3, pp. 363-370, 2011.

[7] F. Alvarez-Guisasola, D. D. Yin, G. Nocea, Y. Qiu, and P. Mavros, "Association of hypoglycemic symptoms with patients' rating of their health-related quality of life state: a cross sectional study," Health and Quality of Life Outcomes, vol. 8, no. 1, p. 86, 2010.

[8] American Diabetes Association, "Standards of Medical Care in Diabetes-2016," Diabetes Care, vol. 39, Supplement 1, pp. S1- 
S111, 2016, https://care.diabetesjournals.org/content/suppl/2015/ 12/21/39.Supplement_1.DC2/2016-Standards-of-Care.pdf.

[9] P. Sakthong, "Measurement of clinical-effect: utility," Journal of the Medical Association of Thailand, vol. 91, Supplement 2, pp. S43-S52, 2008.

[10] The EuroQol Group, "EuroQol - a new facility for the measurement of health-related quality of life," Health Policy, vol. 16, no. 3, pp. 199-208, 1990.

[11] P. Dolan, C. Gudex, P. Kind, and A. Williams, A Social Tariff for EuroQol: Results from a UK General Population Survey, Working Papers 138chedp, Centre for Health Economics, University of York, 1995.

[12] M. J. Atkinson, A. Sinha, S. L. Hass et al., "Validation of a general measure of treatment satisfaction, the treatment satisfaction questionnaire for medication (TSQM), using a national panel study of chronic disease," Health and Quality of Life Outcomes, vol. 2, no. 1, p. 12, 2004.

[13] D. J. Cox, A. Irvine, L. Gonder-Frederick, G. Nowacek, and J. Butterfield, "Fear of hypoglycemia: quantification, validation, and utilization," Diabetes Care, vol. 10, no. 5, pp. 617$621,1987$.

[14] L. A. Gonder-Frederick, K. M. Schmidt, K. A. Vajda et al., "Psychometric properties of the hypoglycemia fear survey-II for adults with type 1 diabetes," Diabetes Care, vol. 34 , no. 4 , pp. 801-806, 2011.

[15] R. W. Grant, N. G. Devita, D. E. Singer, and J. B. Meigs, "Improving adherence and reducing medication discrepancies in patients with diabetes," The Annals of Pharmacotherapy, vol. 37, no. 7-8, pp. 962-969, 2003.

[16] S. P. Chan, L. N. Ji, W. Nitiyanant, S. H. Baik, and W. H. H. Sheu, "Hypoglycemic symptoms in patients with type 2 diabetes in Asia-Pacific-real-life effectiveness and care patterns of diabetes management: the RECAP-DM study," Diabetes Research and Clinical Practice, vol. 89, no. 2, pp. e30-e32, 2010.

[17] M. A. Pourhoseingholi, M. Vahedi, and M. Rahimzadeh, "Sample size calculation in medical studies," Gastroenterology and Hepatology From Bed to Bench, vol. 6, no. 1, pp. 14-17, 2013.

[18] J. Textor, J. Hardt, and S. Knüppel, "DAGitty: a graphical tool for analyzing causal diagrams," Epidemiology, vol. 22, no. 5, p. $745,2011$.

[19] E. Marrett, L. Radican, M. J. Davies, and Q. Zhang, “Assessment of severity and frequency of self-reported hypoglycemia on quality of life in patients with type 2 diabetes treated with oral antihyperglycemic agents: a survey study," BMC Research Notes, vol. 4, no. 1, p. 251, 2011.

[20] F. Á. Guisasola, S. T. Povedano, G. Krishnarajah, R. Lyu, P. Mavros, and D. Yin, "Hypoglycaemic symptoms, treatment satisfaction, adherence and their associations with glycaemic goal in patients with type 2 diabetes mellitus: findings from the real-life effectiveness and care patterns of diabetes management (RECAP-DM) study," Diabetes, Obesity \& Metabolism, vol. 10, Supplement 1, pp. 25-32, 2008.

[21] R. E. Davis, M. Morrissey, J. R. Peters, K. Wittrup-Jensen, T. Kennedy-Martin, and C. J. Currie, "Impact of hypoglycaemia on quality of life and productivity in type 1 and type 2 diabetes," Current Medical Research and Opinion, vol. 21, no. 9, pp. 1477-1483, 2005.

[22] C. D. Miller, L. S. Phillips, D. C. Ziemer, D. L. Gallina, C. B. Cook, and I. M. El-Kebbi, "Hypoglycemia in patients with type
2 diabetes mellitus," Archives of Internal Medicine, vol. 161, no. 13, pp. 1653-1659, 2001.

[23] E. Marrett, T. Stargardt, P. Mavros, and C. M. Alexander, "Patient-reported outcomes in a survey of patients treated with oral antihyperglycaemic medications: associations with hypoglycaemia and weight gain," Diabetes, Obesity \& Metabolism, vol. 11, no. 12, pp. 1138-1144, 2009.

[24] H. Gilet, J.-B. Gruenberger, G. Bader, and M. Viala-Danten, "Demonstrating the burden of hypoglycemia on patients' quality of life in diabetes clinical trials: measurement considerations for hypoglycemia," Value in Health, vol. 15, no. 8, pp. 1036-1041, 2012.

[25] L. Shi, H. Shao, Y. Zhao, and N. A. Thomas, "Is hypoglycemia fear independently associated with health-related quality of life?," Health and Quality of Life Outcomes, vol. 12, no. 1, p. 167, 2014.

[26] J. Pattanaphesaj and M. Thavorncharoensap, "Measurement properties of the EQ-5D-5L compared to EQ-5D-3L in the Thai diabetes patients," Health and Quality of Life Outcomes, vol. 13 , no. 1 , p. 14,2015 . 


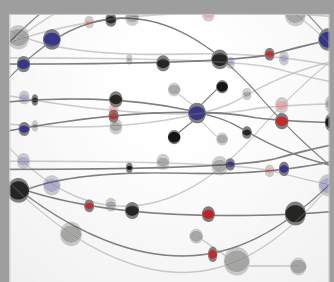

The Scientific World Journal
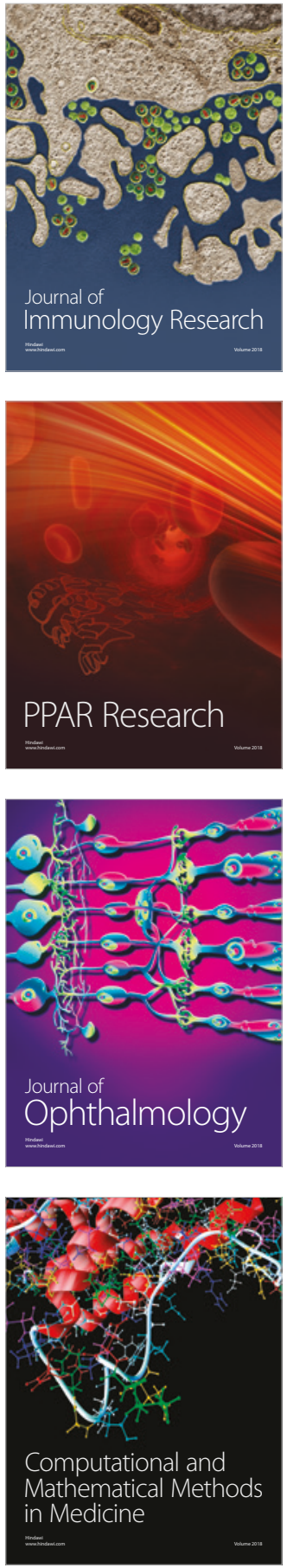

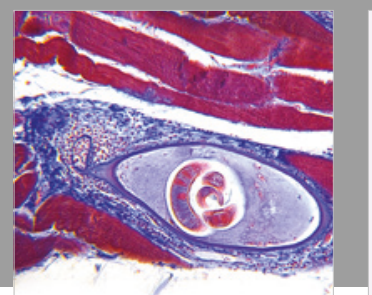

Gastroenterology Research and Practice

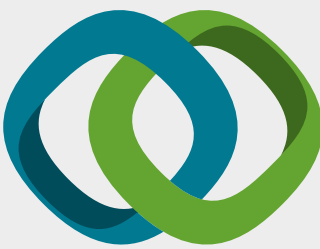

\section{Hindawi}

Submit your manuscripts at

www.hindawi.com
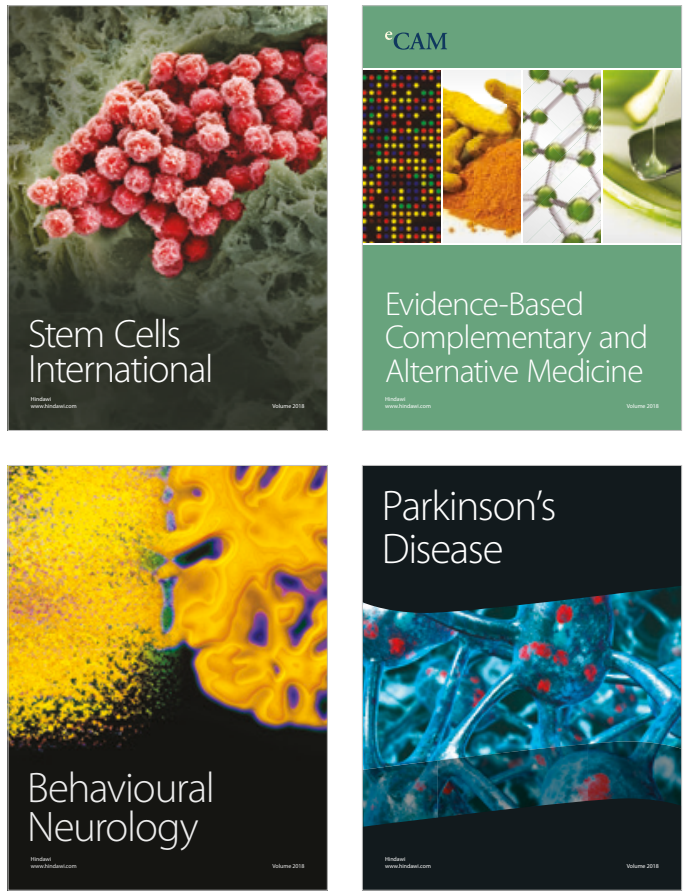

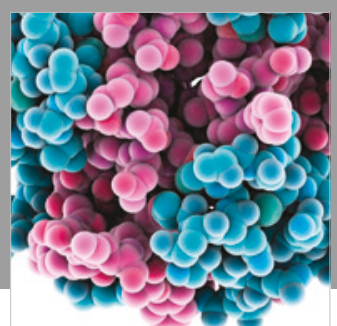

ournal of

Diabetes Research

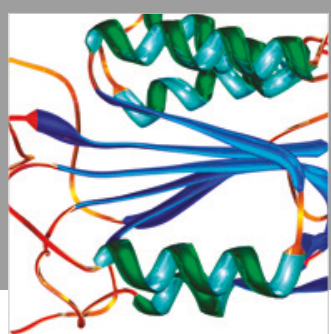

Disease Markers
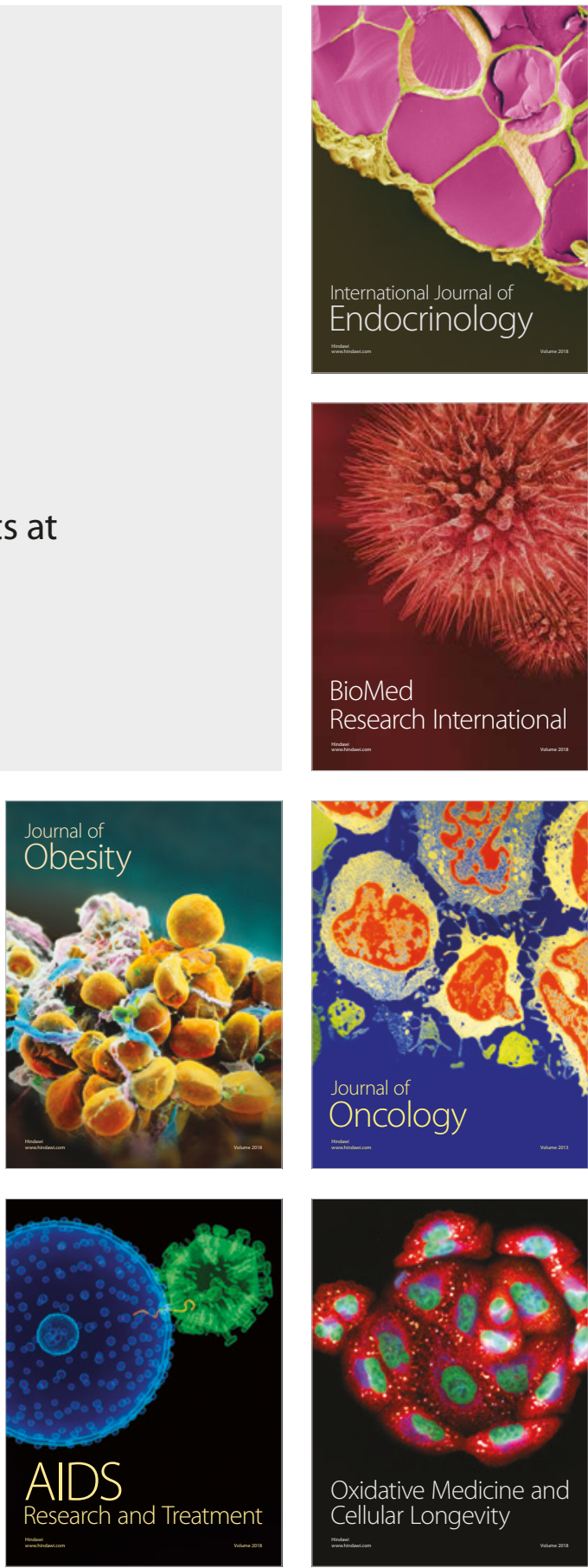